\title{
Prognostic value of cervical nodal necrosis on staging imaging of nasopharyngeal carcinoma in era of intensity-modulated radiotherapy: A systematic review and meta-analysis
}

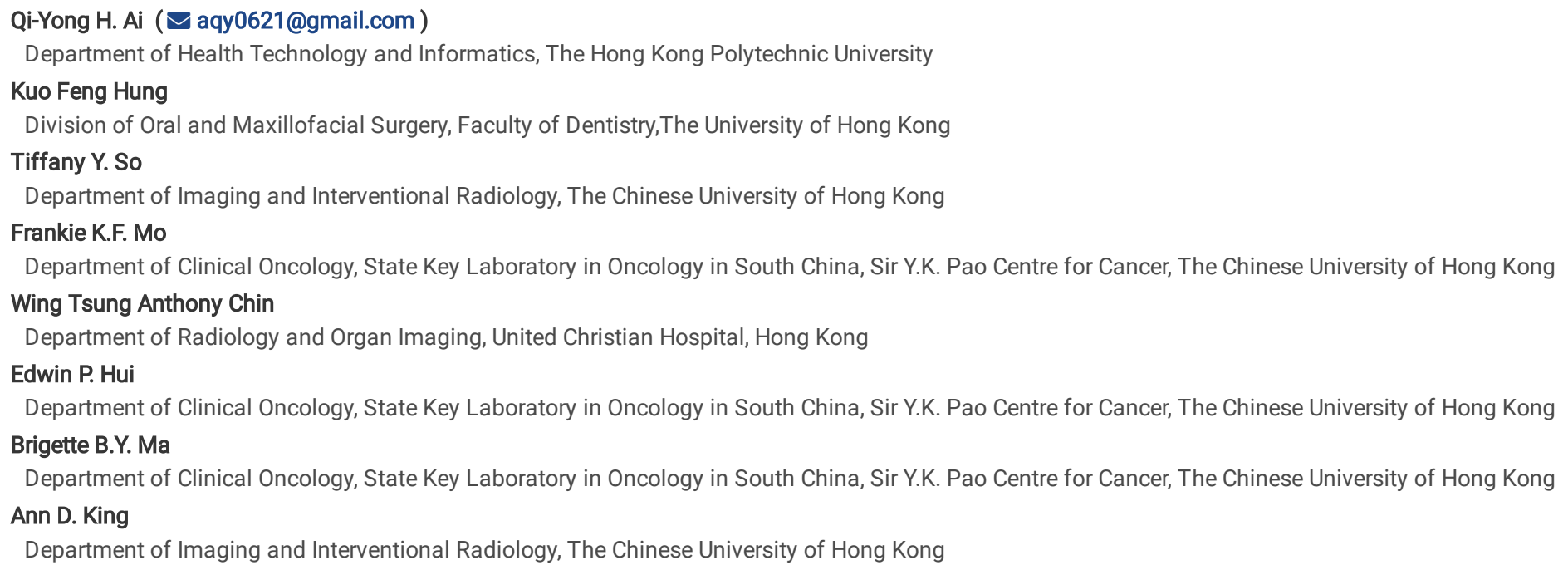




\section{Abstract \\ Purposes}

To systematically review and perform meta-analysis to evaluate the prognostic value of cervical nodal necrosis (CNN) on the staging computed tomography/magnetic resonance imaging (MRI) of nasopharyngeal carcinoma (NPC) in era of intensity-modulated radiotherapy.

\section{Methods}

Literature search through PubMed, EMBASE, and Cochrane Library was conducted. The hazard ratios (HRs) with 95\% confidence intervals (Cls) of CNN for distant metastasis-free survival (DMFS), disease free survival (DFS) and overall survival (OS) were extracted from the eligible studies and meta-analysis was performed to evaluate the pooled $\mathrm{HRs}$ with $95 \% \mathrm{Cl}$.

\section{Results}

Nine studies, which investigated the prognostic values of 6 CNN patterns on MRI were included. Six/9 studies were eligible for meta-analysis, which investigated the CNN presence/absence in any nodal group among 4359 patients. The pooled unadjusted HRs showed that the CNN presence predicted poor DMFS ( $\mathrm{HR}=1.89,95 \% \mathrm{Cl}=1.72-2.08)$, DFS ( $\mathrm{HR}=1.57,95 \% \mathrm{Cl}=1.08-2.26)$, and OS ( $\mathrm{HR}=1.87,95 \% \mathrm{Cl}=1.69-2.06)$. The pooled adjusted $\mathrm{HRs}$ also showed the consistent results for DMFS ( $\mathrm{HR}=1.34,95 \% \mathrm{Cl}=1.17-1.54)$, DFS $(\mathrm{HR}=1.30,95 \% \mathrm{Cl}=1.08-1.56)$, and OS $(\mathrm{HR}=1.61,95 \% \mathrm{Cl}=1.27-2.04)$. Results shown in the other studies analysing different CNN patterns indicated the high grade of CNN predicted poor outcome, but meta-analysis was unable to perform because the heterogeneity of the analysed CNN patterns.

\section{Conclusion}

The CNN observed on the staging MRI is a negative factor for NPC outcome, suggesting that the inclusion of CNN is important in the future survival analysis. However, whether and how should CNN be included in the staging system warrant further evaluation.

\section{Key Points}

1. Cervical nodal necrosis (CNN) is frequently observed in pre-treatment nasopharyngeal carcinoma (NPC);

2. CNN on pre-treatment imaging of NPC is a negative prognosticator of outcome.

3. Inclusion of CNN is critical in the future survival analysis in NPC;

4. It remains unclear whether/how should CNN be included in the staging system.

\section{Introduction}

Nasopharyngeal carcinoma (NPC) is a cancer that derives from the epithelial tissues at the back of the nose and prone to spread outside to the adjacent cervical lymph nodes. About $70 \%$ of patients have metastatic lymph nodes at presentation [1], often at multiple levels in one or both sides of the neck, and these nodes are an important determinant of survival outcome. Imaging, such as computed tomography (CT) and magnetic resonance imaging (MRI), has advantages over clinical examinations in depicting this disease, and has been widely used to evaluate the regional metastatic nodes at presentation [2, 3]. Cervical nodal necrosis (CNN) is one of the nodal characteristics frequently observed in metastatic nodes by CT and MRI [4-6]. Necrosis is usually associated with tissue hypoxia, which a strong negative factor for outcome[7, 8], and so the CNN may have potential to predict outcome. Indeed, some previous studies showed the presence of CNN predicted poor outcome in NPC, but conflicting results also have reported [9-27].

To better understand the prognostic value of the CNN in patients with NPC, this review systematically evaluated and summarised the existing literature on the prognostic value of CNN observed in the staging CT/MRI in patients with NPC treated with intensity-modulated radiotherapy (IMRT). Meta-analysis was performed for the data extracted from the eligible studies. This information may clarify the prognostic value of CNN and help to identify the role of CNN in the cancer staging system.

\section{Methods}

\section{Search strategy}

Methods of the analysis and inclusion criteria were defined in advance, documented, and the proposal was registered at PROSPERO International Prospective Register of Systematic Reviews (ID: CRD42021286561). This study followed the Meta-analyses of Observational Studies in Epidemiology Guidelines [28]. Studies published from the onset of electronic databases up to September 2021 in the PubMed, EMBASE, and Cochrane Library databases were searched with the keywords of "nasopharyngeal AND (carcinoma OR cancer) AND (necrotic OR necrosis) AND (Magnetic Resonance Imaging OR mri OR computed tomography OR CT)". Records were collated Endnote ${ }^{\mathrm{TM}}$ (Version: X7, Clarivate Analytics, New York, NY, USA), and the titles were screened for duplicates. An Institutional Review Board approval or patient written consent was waived as we only used data from the previously published studies.

\section{Selection criteria}


The inclusion criteria were as follows:

(1) Original articles published in English;

(2) Participants: studies with patients with biopsy-proven NPC without distant metastasis and patients who underwent the pre-treatment head and neck staging CT or MR imaging scans;

(3) Comparison: studies that evaluated CNN using CT or MRl;

(4) Outcome: distant metastases-free survival (DMFS) and disease-free survival (DFS) or overall survival.

The exclusion criteria were as follows:

(1) Articles in conference abstracts, case reports, review, and letter to editor formats;

(2) Studies with patients undergone other than IMRT;

(3) Studies not reporting unadjusted or adjusted HR and $95 \% \mathrm{Cl} \mathrm{HR}$ of CNN for DMFS; and

(4) Studies with the patient population overlapped with the previous studies conducted in the same investigated institution for assessing the same CNN pattern.

The records were independently selected by two observers (QYHA and TYS) by assessing the title and then abstracts for the full-text evaluation. Any discrepancy at these steps was resolved by discussion. An additional manual search was performed in the reference lists of the included studies.

\section{Data extraction}

Data extraction was performed independently by the two observers (QYHA and KFH), and the following data were retrieved: first author, journal name, year of publication, city, hospital/institution, patient recruitment period, number of patients, patient treated with chemotherapy, patients with nodes, patients with CNN, age, sex, follow-up time, and edition of the AJCC staging system for NPC, histological type, CNN patterns, group of patients for survival analysis, unadjusted and adjusted HRs with $95 \% \mathrm{Cl}$ of CNN for the survival endpoints. When HRs with $95 \% \mathrm{Cls}$ for outcome endpoints were not provided, we used the methods described by Tierney et al. [29] to estimate them from the data extracted from the respective Kaplan-Meier survival curves using the WebPlotDigitizer [30]. Necrosis was identified as a focal area of high signal intensity on T2-weighted MRI, as an area of non-enhancement on contrast-enhanced T1-weighted MRI, or as an area of low attenuation with or without a surrounding rim of enhancement on contrast-enhanced CT $[4,5]$.

\section{Survival endpoints}

DMFS was set as the primary survival endpoint for the analysis as the distant metastases is the main causes of mortality in NPC. DFS and OS were also included as the additional survival endpoints for the analysis if information was provided. DMFS, DFS and OS were calculated from the date of the diagnosis/start of treatment/end of the treatment to the date of distant metastases, date of any disease recurrence, and date of death, respectively.

\section{Quality assessment}

The Newcastle-Ottawa-Scale (NOS) [31] for cohort studies to assess the quality of the included studies. The NOS evaluated each study in three parts. The NOS score ranges from 0 to 9 , of which 9 indicates the highest quality. The quality assessment was performed by two observers (QYHA and KFH) and discrepancies were solved by discussion with a third observer (TYS).

\section{Statistical analysis}

The inter-observer agreements for the article selection by the titles and abstracts were calculated, respectively, and the Cohen's kappa coefficients were obtained. The meta-analysis was performed for the unadjusted and adjusted HRs of CNN for the survival endpoint(s), respectively. The HRs with $95 \% \mathrm{Cl}$ were pooled with a random- or fixed- effects models. Heterogeneity between studies was assessed using the Cochran Q-statistic and $\mathrm{I}^{2}$ tests. The analysis with an $\mathrm{I}^{2}$ value larger than $50 \%$ was considered as substantial heterogeneity. The fixed-effects model was used if the heterogeneity was not significant $\left(\mathrm{I}^{2}<50 \%\right.$ and $\mathrm{p}$ value $>0.05$ ); otherwise, a random-effects model was applied. To test whether a single study affected the combined HR, a sensitivity analysis was performed. All statistical tests were two-sided, and a p-value of less than 0.05 was considered to indicate a statistically significant difference. Publication bias was tested by Begg's test and Egger's test. For the interpretation of Egger's test, statistical significance was defined as p-value of less than 0.1 . The meta-analysis was performed using Stata IC (Version 16.0, StataCorp LLC, TX, USA).

\section{Results}

\section{Study selection}

Figure 1 shows the flowchart for study selection. A total of 668 potential records were initially identified by searching the electronic databases (Figure 1 ). After the initial exclusion of the duplicates, reviews, conference abstracts, and letters $(n=421)$, and further exclusion of 214 and 6 records after assessing the titles and abstracts, respectively, 27 studies remained for full-text reading. No additional articles were identified through manual search from the reference list of the included studies. The kappa coefficients for the study selection by assessing the titles and abstracts were 0.81 and 0.83 , respectively. Eighteen studies were subsequently excluded due to the specific reasons (Table 1) [9-18, 32-39], leaving 9 studies for the further systematic review and analysis [19-27]. 
Table 1

Numbers and reasons for the excluded articles after full-text review

\begin{tabular}{|c|c|c|}
\hline Reasons to exclude the articles after the full-text review & $\begin{array}{l}\text { Numbers of } \\
\text { studies }\end{array}$ & $\begin{array}{l}\text { Numbers in the } \\
\text { references list }\end{array}$ \\
\hline Patients treated with other than IMRT & 3 & {$[9-11]$} \\
\hline HRs of CNN for DMFS was not performed or unable to calculate & 7 & [12-18] \\
\hline Survival analysis was not performed for CNN & 2 & {$[32,33]$} \\
\hline $\mathrm{CNN}+$ necrotic primary tumour as one variable was assessed & 1 & [34] \\
\hline $\begin{array}{l}\text { Studies conducted by the same institution with overlapping patient recruitment periods and analysis } \\
\text { performed for the same CNN pattern }\end{array}$ & 5 & [35-39] \\
\hline
\end{tabular}

\section{Characteristics of the eligible studies}

Characteristics of the included studies are shown in Table 2. The included studies were conducted by 5 institutions and the CNN were all evaluated on MRI. Three conducted by the same institution were included because these studies performed analysis to evaluate prognostic values of different CNN patterns [2527]. Analysis was performed to evaluate prognostic values of $6 \mathrm{CNN}$ patterns, which included (1) CNN presence/absence in any nodal group ( $\mathrm{n}=6$ ) [19-24],

(2) CNN presence/absence in retropharyngeal nodes (RPNs) ( $n=1)$ [25], (3) CNN grades ( $n=1)$ [26], (4) CNN laterality ( $n=1)$ [27], (5) total CNN volume [23], and (6) maximum percentage of nodal necrotic volume of one single node (necrosis\%) [23]. According to the NOS criteria, the quality of the eligible studies ranged from 7 to 9 with a median score of 8 (Table 3). 
Table 2

Characteristics of the eligible articles

\begin{tabular}{|c|c|c|c|c|c|c|c|c|c|c|c|}
\hline $\begin{array}{l}\text { First } \\
\text { author }\end{array}$ & $\begin{array}{l}\text { Year of } \\
\text { Publication }\end{array}$ & City & $\begin{array}{l}\text { Patient } \\
\text { recruitment } \\
\text { period }\end{array}$ & $\begin{array}{l}\text { Total } \\
\text { patients }\end{array}$ & $\begin{array}{l}\text { Patient } \\
\text { treated with } \\
\text { chemotherapy }\end{array}$ & $\begin{array}{l}\text { Patient } \\
\text { with } \\
\text { nodes }\end{array}$ & $\begin{array}{l}\text { Patient with } \\
\text { CNN }\end{array}$ & $\begin{array}{l}\text { Imaging } \\
\text { modality }\end{array}$ & $\begin{array}{l}\text { Numbers of } \\
\text { undifferentiated } \\
\text { + non- } \\
\text { keratinising } \\
\text { types }\end{array}$ & $\begin{array}{l}\text { AJCC } \\
\text { /UICC } \\
\text { Edition }\end{array}$ & $\begin{array}{l}\text { Me } \\
\text { foll } \\
\text { tim } \\
\text { (ral } \\
\text { (me }\end{array}$ \\
\hline \multicolumn{12}{|c|}{ CNN presence/absence in any nodal group } \\
\hline Li [19] & 2013 & Guangzhou & $2003-2007$ & 749 & 535 & 565 & 142 & MRI & 744 & 7th & 60. \\
\hline $\begin{array}{l}\text { Zhang } \\
\text { [20] }\end{array}$ & 2017 & Guangzhou & 2009-2012 & 1302 & 1193 & 1302 & 448 & MRI & 1294 & 7th & $\begin{array}{l}47 . \\
(1 . \approx\end{array}$ \\
\hline $\begin{array}{l}\text { Zhou } \\
\text { [21] }\end{array}$ & 2018 & Shanghai & $2010-2011$ & 354 & 300 & 320 & 143 & MRI & 353 & 7th & $\begin{array}{l}63 \\
\text { (No } \\
\text { me }\end{array}$ \\
\hline $\begin{array}{l}\text { Feng } \\
\text { [22] }\end{array}$ & 2019 & Hangzhou & 2007-2012 & 616 & 601 & 616 & 235 & MRI & 612 & 8th & $\begin{array}{l}62 . \\
(3.4\end{array}$ \\
\hline Ai [23] & 2019 & Hong Kong & $2005-2012$ & 546 & 382 & 404 & 153 & MRI & 544 & 8th & 82: \\
\hline $\begin{array}{l}\mathrm{Xu} \\
{[24]}\end{array}$ & 2021 & Xi'an & $2006-2018$ & 792 & 744 & 687 & 501 & MRI & 789 & 7th & $\begin{array}{l}\text { 46.: } \\
(1 . \approx\end{array}$ \\
\hline \multicolumn{12}{|c|}{ CNN presence/absence in retropharyngeal nodes } \\
\hline $\begin{array}{l}\text { Tang } \\
{[25]}\end{array}$ & 2014 & Guangzhou & $2003-2007$ & 749 & 535 & 565 & $\begin{array}{l}64 / 484 \\
\text { (RPN+) }\end{array}$ & MRI & 744 & 7th & $\begin{array}{l}81 \\
(3-1\end{array}$ \\
\hline \multicolumn{12}{|c|}{ CNN grades } \\
\hline \multirow{2}{*}{$\begin{array}{l}\text { Zhang } \\
\text { [26] }\end{array}$} & 2017 & Guangzhou & 2009-2012 & 1423 & 1310 & 1423 & Grade 1: 213 & MRI & 1415 & 7th & 48. \\
\hline & & & & & & & Grade 2: 257 & & & & $(1 . \Xi$ \\
\hline \multicolumn{12}{|c|}{ CNN laterality } \\
\hline \multirow[t]{2}{*}{$\begin{array}{l}\text { Xie } \\
{[27]}\end{array}$} & 2020 & Guangzhou & 2010-2013 & 733 & 634 & 559 & $\begin{array}{l}\text { No/unilateral } \\
\text { CNN: } 692\end{array}$ & MRI & 728 & 8th & \multirow{2}{*}{$\begin{array}{l}62 \\
(1.4\end{array}$} \\
\hline & & & & & & & $\begin{array}{l}\text { Bilateral } \\
\text { CNN: } 41\end{array}$ & & & & \\
\hline
\end{tabular}

$\mathrm{CNN}=$ cervical nodal necrosis, $\mathrm{AJCC} / \mathrm{UICC}=$ American Joint of Cancer Committee/ Union for International Cancer Control, $\mathrm{N}+\mathrm{group}=$ patients with metastatic I =retropharyngeal node, MRI =magnetic resonance imaging 
The Newcastle-Ottawa Scale(NOS) quality assessment of the eligible studies in the meta-analysis

\begin{tabular}{|c|c|c|c|c|c|c|c|c|c|}
\hline \multirow{4}{*}{$\begin{array}{l}\text { First } \\
\text { author }\end{array}$} & \multirow{4}{*}{$\begin{array}{l}\text { Year of } \\
\text { publication }\end{array}$} & \multicolumn{4}{|l|}{ Selection } & \multirow{2}{*}{$\begin{array}{l}\text { Comparability } \\
\text { Comparability } \\
\text { of cohorts on } \\
\text { the basis of } \\
\text { the design or } \\
\text { analysis }\end{array}$} & \multicolumn{3}{|l|}{ Outcome } \\
\hline & & $\begin{array}{l}\text { Representativeness } \\
\text { of the exposed } \\
\text { cohort } \\
(0-1)\end{array}$ & $\begin{array}{l}\text { Selection } \\
\text { of the } \\
\text { non- } \\
\text { exposed } \\
\text { cohort }\end{array}$ & $\begin{array}{l}\text { Ascertainment } \\
\text { of exposure } \\
(0-1)\end{array}$ & $\begin{array}{l}\text { Demonstration } \\
\text { that outcome } \\
\text { of interest was } \\
\text { not present at } \\
\text { start of study }\end{array}$ & & $\begin{array}{l}\text { Assessment } \\
\text { of outcome } \\
(0-1)\end{array}$ & $\begin{array}{l}\text { Was } \\
\text { follow-up } \\
\text { long } \\
\text { enough } \\
\text { for }\end{array}$ & $\begin{array}{l}\text { Adequacy } \\
\text { of follow } \\
\text { up of } \\
\text { cohorts }\end{array}$ \\
\hline & & & $(0-1)$ & & $(0-1)$ & $(0-2)$ & & to occur & \\
\hline & & & & & & & & $(0-1)$ & \\
\hline
\end{tabular}

CNN presence/absence in any nodal group

\begin{tabular}{|c|c|c|c|c|c|c|c|c|c|}
\hline Li [19] & 2013 & 1 & 1 & 0 & 1 & 2 & 1 & 1 & 1 \\
\hline $\begin{array}{l}\text { Zhang } \\
\text { [20] }\end{array}$ & 2017 & 1 & 1 & 1 & 1 & 2 & 0 & 1 & 1 \\
\hline $\begin{array}{l}\text { Zhou } \\
\text { [21] }\end{array}$ & 2018 & 1 & 1 & 1 & 1 & 2 & 0 & 1 & 1 \\
\hline $\begin{array}{l}\text { Feng } \\
\text { [22] }\end{array}$ & 2019 & 1 & 1 & 1 & 1 & 2 & 0 & 1 & 1 \\
\hline Ai [23] & 2019 & 1 & 1 & 1 & 1 & 2 & 1 & 1 & 1 \\
\hline $\begin{array}{l}X u \\
{[24]}\end{array}$ & 2021 & 1 & 1 & 0 & 1 & 2 & 0 & 1 & 1 \\
\hline
\end{tabular}

CNN presence/absence in retropharyngeal nodes

$\begin{array}{lllllllll}\text { Tang } & 2020 & 1 & 1 & 0 & 1 & 2 & 1 & 1\end{array}$

[25]

CNN grades

\begin{tabular}{|c|c|c|c|c|c|c|c|c|c|}
\hline $\begin{array}{l}\text { Zhang } \\
\text { [26] }\end{array}$ & 2021 & 1 & 1 & 1 & 1 & 2 & 1 & 1 & 1 \\
\hline \multicolumn{10}{|c|}{ CNN laterality } \\
\hline $\begin{array}{l}\text { Xie } \\
\text { [27] }\end{array}$ & 2021 & 1 & 1 & 1 & 1 & 2 & 1 & 1 & 1 \\
\hline
\end{tabular}

Three studies were excluded from the meta-analysis due to the great heterogeneity of the analysed CNN patterns or the limited studies for evaluating each of the CNN patterns. Meta-analysis therefore was only eligible to perform in 6 studies which evaluated the prognostic value of CNN presence/absence in any nodal group [19-24]. The eligible studies for meta-analysis were published over 9 years (2013[19]- 2021 [24]) (Table 2). Patient recruitment ranged from 2003 to 2018 with the follow-up periods ranged from 1.3 to 150 months (Table 2). The total patient number extracted from these studies was 4359 (range 354 1302) with ages ranging from 12 to 90 years, of which $3216(73.8 \%)$ were male and $1143(26.2 \%)$ were female. Metastatic cervical nodes were observed in 3894 patients, of which CNN was observed in 1622/3894 (41.7\%) patients (Table 2). Over $99 \%$ of patients had undifferentiated carcinoma or non-keratinising carcinoma (Table 2).

\section{Prognostic value of the CNN presence/absence}

Of the 6 studies that are eligible for meta-analysis, 4 studies performed the survival analysis in patients with metastatic nodes ( $\mathrm{N}+$ group), and 2 in all patients (Table 2). The unadjusted and adjusted HRs of CNN were reported for DMFS in 5 and 5 studies respectively, for DFS in 3 and 4 studies, respectively, and for OS in 3 and 4 studies, respectively (Figure 2-4).

The pooled unadjusted HRs showed that the presence of CNN predicted poor DMFS (HR=1.89, 95\%Cl $\left.=1.72-2.08, I^{2}=44.8 \%, p=0.123\right)(F i g u r e 2)$, DFS $(H R$ $\left.=1.57,95 \% \mathrm{Cl}=1.08-2.26, \mathrm{I}^{2}=71.1 \%, \mathrm{p}=0.063\right)$ (Figure 3$)$, and OS $\left(\mathrm{HR}=1.87,95 \% \mathrm{Cl}=1.69-2.06, \mathrm{I}^{2}=9.1 \%, \mathrm{p}=0.333\right)($ Figure 4$)$. The pooled adjusted $\mathrm{HRs}$ also showed the consistent results for DMFS $\left(\mathrm{HR}=1.34,95 \% \mathrm{Cl}=1.17-1.54, \mathrm{I}^{2}=0.0 \%, \mathrm{p}=0.426\right)($ Figure 2$), \mathrm{DFS}\left(\mathrm{HR}=1.30,95 \% \mathrm{Cl}=1.08-1.56, \mathrm{I}^{2}=12.7 \%\right.$, $\mathrm{p}=0.318$ ) (Figure 3$)$, and OS ( $\left.\mathrm{HR}=1.61,95 \% \mathrm{Cl}=1.27-2.04, \mathrm{I}^{2}=0.4 \%, \mathrm{p}=0.390\right)$ (Figure 4). The sensitivity tests of the unadjusted and adjusted $\mathrm{HRs}$ for the survival endpoints in the meta-analysis are shown in Figure 5. Subgroup meta-analysis was performed for the adjusted HRs to further evaluate the prognostic value of CNN presence/absence in patients with metastatic nodes ( + group). Results showed that the presence of CNN predicted poor DMFS (HR $=1.56$, $\left.95 \% \mathrm{Cl}=1.25-1.95, \mathrm{I}^{2}=0.0 \%, \mathrm{p}=0.837\right)($ Figure 2$), \mathrm{DFS}\left(\mathrm{HR}=1.43,95 \% \mathrm{Cl}=1.05-1.95, \mathrm{I}^{2}=40.1 \%, \mathrm{p}=0.196\right)($ Figure 3$)$, and OS $(\mathrm{HR}=1.55,95 \% \mathrm{Cl}=1.21-$ $2.00, I^{2}=12.3 \%, p=0.320$ ) (Figure 4).

The Begg's and Egger's tests showed that no potential publication bias were observed in the meta-analysis (Begg's, $p=0.211$ to $>0.999 ;$ Egger's test, $p=0.132$ to 0.905 ) except for that of the unadjusted HRs for DMFS (Egger's test, $p=0.014$ ).

Prognostic values of other CNN patterns 
Tang et al[25] evaluated the prognostic value of the presence of CNN in the retropharyngeal nodes (RPNs) in patients with metastatic nodes (N+ group) showing the presence of CNN independently predicted poor DMFS ( $\mathrm{HR}=1.75,95 \% \mathrm{Cl}=1.10-2.79)$, and DFS $(\mathrm{HR}=1.80,95 \% \mathrm{Cl}=1.21-2.65)$. Zhang et al[26] classified patients with nodes into 3 grades of necrosis (grade 0: no necrotic area; grade 1: any node with necrotic area of $\leq 33 \%$; and grade 2 : any node with necrotic area of $>33 \%$ ) and added the necrosis grades as the continuous variable to the survival analysis. Results showed that patients with higher necrosis grades independently predicted poorer DMFS $(\mathrm{HR}=1.36,95 \% \mathrm{Cl}=1.14-1.63)$, DFS $(\mathrm{HR}=1.38,95 \% \mathrm{Cl}=1.21-1.59)$, and $0 \mathrm{~S}(\mathrm{HR}=1.36,95 \% \mathrm{Cl}=1.13-2.45)$. Xie et al[27] reported patients with bilateral CNN independently predicted poorer DMFS ( $\mathrm{HR}=2.10,95 \% \mathrm{Cl}=1.10-2.40)$ compared to those with unilateral CNN or without CNN. The study conducted by Ai et al.[23] also quantitatively evaluated the total CNN volume and necrosis\% showing necrosis\%, but not the total CNN volume, was a factor for predicting DMFS ( $\mathrm{HR}=3.03,95 \% \mathrm{Cl}=1.242-7.397)$ and $\mathrm{OS}(\mathrm{HR}=3.09,95 \% \mathrm{Cl}=1.482-6.431)$; while necrosis\% was not an independent factor to predict outcome when other confounding factors were added to the multivariate analysis.

\section{Discussion}

The current review article systematically investigated the studies that evaluated the prognostic values of CNN in patients with NPC, and performed metaanalysis to assess the pooled HRs of CNN presence in any nodal group for DMFS, DFS and OS in 4359 patients. Although over 20 studies have investigated the prognostic value of CNN in patients with NPC, more than half of these studies were excluded for meta-analysis due to insufficient information reported. According the NOS assessment, all eligible studies were scored from 7-9, indicating the data extracted from the studies are reliable. Results from the metaanalysis in 6 studies showed that the CNN presence in any nodal group predicted poor DMFS, DFS, and OS with the pooled adjusted HRs ranging from 1.34 to 1.61 when analysis included all patients, and from 1.43 to 1.56 when analysis only included patients with metastatic nodes ( $N+$ group). Four eligible studies evaluated the prognostic values of five different CNN patterns, which also showed the CNN is a negative factor for outcome.

With the introduction of IMRT to NPC, primary tumour has been well controlled with a local relapse rate of less than $10 \%$, but distant metastases remain problematic and is now the main cause of mortality [40,41]. More and more studies have found that the presence of nodal metastases is one of the key factors that predict patients at risk of distant metastases. These studies have gone further to investigate the prognostic values of nodal characteristics in detail [42-46]. Necrosis is easily observed in metastatic nodes from NPC but very rarely in primary tumour (about 2\%) on the pre-treatment staging MRI [34]. Tang et al [25] reported that CNN was observed in 13\% patients with metastatic retropharyngeal nodes, and the current study showed that the incidence of CNN rate was about $42 \%$ in patients with metastatic nodes. In the current study, meta-analysis was firstly performed for the unadjusted HRs of CNN, and the results showed that the presence of $\mathrm{CNN}$ in any nodal group indeed was a factor to predict poor outcome endpoints. This is expected because necrosis in the pre-treatment malignant tissues commonly indicates the hypoxia of the tissues, and previous studies have also shown that hypoxic malignant tissues are resistant to the treatment $[7,8]$. Additionally, meta-analysis was further performed for the adjusted HRs of CNN, and the results indicated that the presence of CNN in any nodal group remained strong to independently predict outcome endpoints after adjusting the other confounding factors.

Two studies further qualitatively evaluated the role of CNN burden showing that patients who had grade 2 CNN (with a necrotic area over 33\% in any node)[26] or with bilateral CNN[27] had poor outcome. A quantitative analysis[23] showed that necrosis\% was a factor that predicted poor outcome. These results indicated the high CNN burden in the nodes may reflect the serve hypoxia in the tumour, thus predicted the high risk of disease recurrence. However, whether the grade of CNN burden was an independent predictor for outcome is still unclear as the multivariate analysis in these studies showed conflicting results while meta-analysis was unlikely to be performed due to the great heterogeneity in the CNN patterns analysed in these studies.

Some studies have proposed their modified criteria by incorporating the CNN to the current staging system showing the improvement in the prognostic performance [10, 21, 22]. However, the differences in the pooled HRs of the CNN and extranodal extension (ENE) reported in a previous meta-analysis (the pooled HRs of the ambiguous ENE ranged from 2.62-3.14) [46]indicates that the modification for the cancer staging system should take into account the role of other nodal characteristics. Although the present study could not answer the question whether and how CNN should be included in the current staging system, results indicate that the inclusion of CNN is critical in the future studies aiming to investigate the prognostic values of nodal characteristics in NPC.

This study has some limitations that may result in the inherent heterogeneity and the publication bias. First, all studies included in this systematic review were retrospective studies. Due to the retrospective nature, the concerns regarding the risk of bias in the included studies could not be avoid. Second, the unadjusted $\mathrm{HR}$ and $95 \% \mathrm{Cl}$ of HRs in one study was extracted and calculated from the survival curves [20]; Third, the potential reasons that resulted in the heterogeneity of the meta-analysis was not able to be analysed due to the limited numbers of the eligible studies. Furthermore, the publication bias could not be excluded as studies with positive results were more likely to be accepted for publication.

\section{Conclusion}

Results from the current study showed that the presence of CNN in any nodal group observed on the pre-treatment staging MRI is a negative factor for DMFS, DFS, and OS in patients with NPC. Although it remains to be defined whether and how should CNN be included in the staging system, the inclusion of CNN is critical in the future survival analysis in NPC.

\section{Abbreviations}

$95 \% \mathrm{Cl}$

95\% confidence interval

CNN

Cervical nodal necrosis

CT 
computed tomography

DFS

disease free survival

DMFS

distant metastases free survival

HR

hazard ratio

MRI

magnetic resonance imaging

NPC

nasopharyngeal carcinoma

NOS

Newcastle-Ottawa-Scale

OS

overall survival

\section{References}

1. Ho FC, Tham IW, Earnest A, et al (2012) Patterns of regional lymph node metastasis of nasopharyngeal carcinoma: A meta-analysis of clinical evidence. BMC Cancer 12:98.

2. King AD, Bhatia KSS (2010) Magnetic resonance imaging staging of nasopharyngeal carcinoma in the head and neck. World journal of radiology 2:159

3. Abdel Khalek Abdel Razek A, King A (2012) MRI and CT of nasopharyngeal carcinoma. AJR American journal of roentgenology 198:11-8.

4. Chong VF, Fan YF, Khoo JB (1996) MRI features of cervical nodal necrosis in metastatic disease. Clinical radiology 51:103-9

5. King AD, Tse GMK, Ahuja AT, et al (2004) Head and Neck Imaging Radiology Necrosis in Metastatic Neck Nodes: Diagnostic Accuracy of CT, MR Imaging, and US. Radiology 230:720-726

6. Anzai Y, Brunberg JA, Lufkin RB (1997) Imaging of nodal metastases in the head and neck. Journal of Magnetic Resonance Imaging 7:774-783.

7. Hui EP, Chan ATC, Pezzella F, et al (2002) Coexpression of hypoxia-inducible factors 1alpha and 2alpha, carbonic anhydrase IX, and vascular endothelial growth factor in nasopharyngeal carcinoma and relationship to survival. Clinical Cancer Research 8:2595-2604

8. Hong B, Lui VWY, Hashiguchi M, et al (2013) Targeting tumor hypoxia in nasopharyngeal carcinoma. Head \& neck 35:133-145

9. Chua DT, Sham JS, Kwong DL, et al (1997) Evaluation of cervical nodal necrosis in nasopharyngeal carcinoma by computed tomography: incidence and prognostic significance. Head \& neck 19:266-275

10. Lan M, Huang Y, Chen CY, et al (2015) Prognostic Value of Cervical Nodal Necrosis in Nasopharyngeal Carcinoma: Analysis of 1800 Patients with Positive Cervical Nodal Metastasis at MR Imaging. Radiology 276:536-544.

11. Lu L, Wei X, Li YH, Li WB (2017) Sentinel node necrosis is a negative prognostic factor in patients with nasopharyngeal carcinoma: A magnetic resonance imaging study of 252 patients. Current Oncology 24:e220-e225.

12. Chen B, Zhan Z, Pan J, et al (2021) Re-evaluation of the prognostic significance of retropharyngeal node metastasis in nasopharyngeal carcinoma patients treated with intensity-modulated radiotherapy. Asia-Pacific Journal of Clinical Oncology 1-9.

13. Guo Q, Pan J, Zong J, et al (2015) Suggestions for Lymph Node Classification of UICC/AJCC Staging System. Medicine $94:$ e808.

14. Chen FP, Zhou GQ, Qi ZY, et al (2017) Prognostic value of cervical nodal tumor volume in nasopharyngeal carcinoma: Analysis of 1230 patients with positive cervical nodal metastasis. PLoS ONE 12:1-13.

15. Mao YP, Liang SB, Liu LZ, et al (2008) The N staging system in nasopharyngeal carcinoma with radiation therapy oncology group guidelines for lymph node levels based on magnetic resonance imaging. Clinical Cancer Research 14:7497-7503.

16. Ou X, Miao Y, Wang X, et al (2017) The feasibility analysis of omission of elective irradiation to level IB lymph nodes in low-risk nasopharyngeal carcinoma based on the 2013 updated consensus guideline for neck nodal levels. Radiation Oncology 12:137.

17. Luo Y, Ren J, Zhou P, et al (2016) Cervical nodal necrosis is an independent survival predictor in nasopharyngeal carcinoma: an observational cohort study. Onco Targets and Therapy 9:6775-6783

18. Ting Y, Chee J, Charn TC, et al (2017) Prognostic significance of cystic lymph nodal metastasis in nasopharyngeal carcinoma. Head and Neck 39:18321839.

19. Li W-F, Sun Y, Mao Y-P, et al (2013) Proposed lymph node staging system using the International Consensus Guidelines for lymph node levels is predictive for nasopharyngeal carcinoma patients from endemic areas treated with intensity modulated radiation therapy. International journal of radiation oncology, biology, physics 86:249-56

20. Zhang LL, Zhou GQ, Li YY, et al (2017) Combined prognostic value of pretreatment anemia and cervical node necrosis in patients with nasopharyngeal carcinoma receiving intensity-modulated radiotherapy: A large-scale retrospective study. Cancer Medicine 6:2822-2831.

21. Zhou X, Ou X, Yang Y, et al (2018) Quantitative Metastatic Lymph Node Regions on Magnetic Resonance Imaging Are Superior to AJCC N Classification for the Prognosis of Nasopharyngeal Carcinoma. Journal of Oncology 2018: 9172585.

22. Feng Y, Cao C, Hu Q, Chen X (2019) Prognostic value and staging classification of lymph nodal necrosis in nasopharyngeal carcinoma after intensitymodulated radiotherapy. Cancer Research and Treatment 51:1222-1230. 
23. Ai QY, King AD, Poon DMC, et al (2019) Extranodal extension is a criterion for poor outcome in patients with metastatic nodes from cancer of the nasopharynx. Oral Oncology 88:124-130.

24. Xu M, Zang J, Luo S, et al (2021) Long-term survival outcomes and adverse effects of nasopharyngeal carcinoma patients treated with IMRT in a nonendemic region: A population-based retrospective study. BMJ Open 11:1-11.

25. Tang LL, Guo R, Zhou G, et al (2014) Prognostic value and staging classification of retropharyngeal lymph node metastasis in nasopharyngeal carcinoma patients treated with intensity-modulated radiotherapy. PLoS ONE 9:1-8.

26. Zhang L-L, Li J-X, Zhou G-Q, et al (2017) Influence of Cervical Node Necrosis of Different Grades on the Prognosis of Nasopharyngeal Carcinoma Patients Treated with Intensity-Modulated Radiotherapy. Journal of Cancer 8:959-966.

27. Xie C, Li H, Yan Y, et al (2020) A Nomogram for Predicting Distant Metastasis Using Nodal-Related Features Among Patients With Nasopharyngeal Carcinoma. Frontiers in Oncology 10:616.

28. Stroup DF, Berlin JA, Morton SC, et al (2000) Meta-analysis of Observational Studies in Epidemiology: A Proposal for Reporting. JAMA 283:2008-2012

29. Tierney JF, Stewart LA, Ghersi D, et al (2007) Practical methods for incorporating summary time-to-event data into meta-analysis. Trials 8:16.

30. Rohatgi A (2021) WebPlotDigitizer: Version 4.5. In: https://automeris.io/WebPlotDigitizer

31. Wells G, Shea B, O'Connell D, et al The Newcastle-Ottawa Scale (NOS) for assessing the quality of nonrandomised studies in meta-analyses. In: Http://www.ohri.ca/programs/clinical_epidemiology/oxford.asp

32. Lan M, Chen C, Huang Y, et al (2017) Neoadjuvant chemotherapy followed by concurrent chemoradiotherapy versus concurrent chemoradiotherapy alone in nasopharyngeal carcinoma patients with cervical nodal necrosis. Scientific Reports 7:42624.

33. Yeh SA, Tang Y, Lui CC, et al (2005) Treatment outcomes and late complications of 849 patients with nasopharyngeal carcinoma treated with radiotherapy alone. International Journal of Radiation Oncology Biology Physics 62:672-679.

34. Liang SB, Chen LS, Yang XL, et al (2021) Influence of tumor necrosis on treatment sensitivity and long-term survival in nasopharyngeal carcinoma. Radiotherapy and Oncology 155:219-225.

35. Du YY, Luo DH, Sun XS, et al (2019) Combining pretreatment plasma Epstein-Barr virus DNA level and cervical node necrosis improves prognostic stratification in patients with nasopharyngeal carcinoma: A cohort study. Cancer Medicine 8:6841-6852.

36. Chen X, Cao X, Jing B, et al (2020) Prognostic and Treatment Guiding Significance of MRI-Based Tumor Burden Features and Nodal Necrosis in Nasopharyngeal Carcinoma. Frontiers in Oncology 10:1-11.

37. Liu K, Lin S, Ke L, et al (2020) Prognostic value and the potential role of treatment options for cervical lymph node necrosis in nasopharyngeal carcinoma. Oral Oncology 109:104864.

38. Tian Y-M, Zeng L, Lan Y-H, et al (2021) The Value of Cervical Node Features in Predicting Long-Term Survival of Nasopharyngeal Carcinoma in the Intensity-Modulated Radiotherapy Era. Cancer Management and Research Volume 13:4899-4909.

39. Niu X, Xue F, Liu P, et al (2021) Long-term outcomes of induction chemotherapy followed by intensity-modulated radiotherapy and adjuvant chemotherapy in nasopharyngeal carcinoma patients with N3 disease. Translational Oncology 14(12):101216

40. Chen YP, Chan ATC, Le QT, et al (2019) Nasopharyngeal carcinoma. The Lancet 394:64-80.

41. Wong KCW, Hui EP, Lo KW, et al (2021) Nasopharyngeal carcinoma: an evolving paradigm. Nature Reviews Clinical Oncology 18:679-695.

42. Ma H, Qiu Y, Li H, et al (2021) Prognostic Value of Nodal Matting on MRI in Nasopharyngeal Carcinoma Patients. Journal of Magnetic Resonance Imaging 53:152-164.

43. Setakornnukul J, Thephamongkhol K, Chaysiri $P$ (2020) Added value of metastatic cervical lymph node group V in nodal staging of nasopharyngeal cancer. Head and Neck 42:2801-2810.

44. Ai QY, King AD, Mo FKF, et al (2018) Staging nodal metastases in nasopharyngeal carcinoma: which method should be used to measure nodal dimension on MRI? Clinical Radiology 73:640-646.

45. Ai Q-Y, King AD, Mo FKF, et al (2017) Prediction of distant metastases from nasopharyngeal carcinoma: Improved diagnostic performance of MRI using nodal volume in N1 and N2 stage disease. Oral Oncology 69:74-79.

46. Tsai TY, Chou YC, Lu YA, et al (2021) The prognostic value of radiologic extranodal extension in nasopharyngeal carcinoma: Systematic review and metaanalysis. Oral Oncology 122:105518.

\section{Figures}




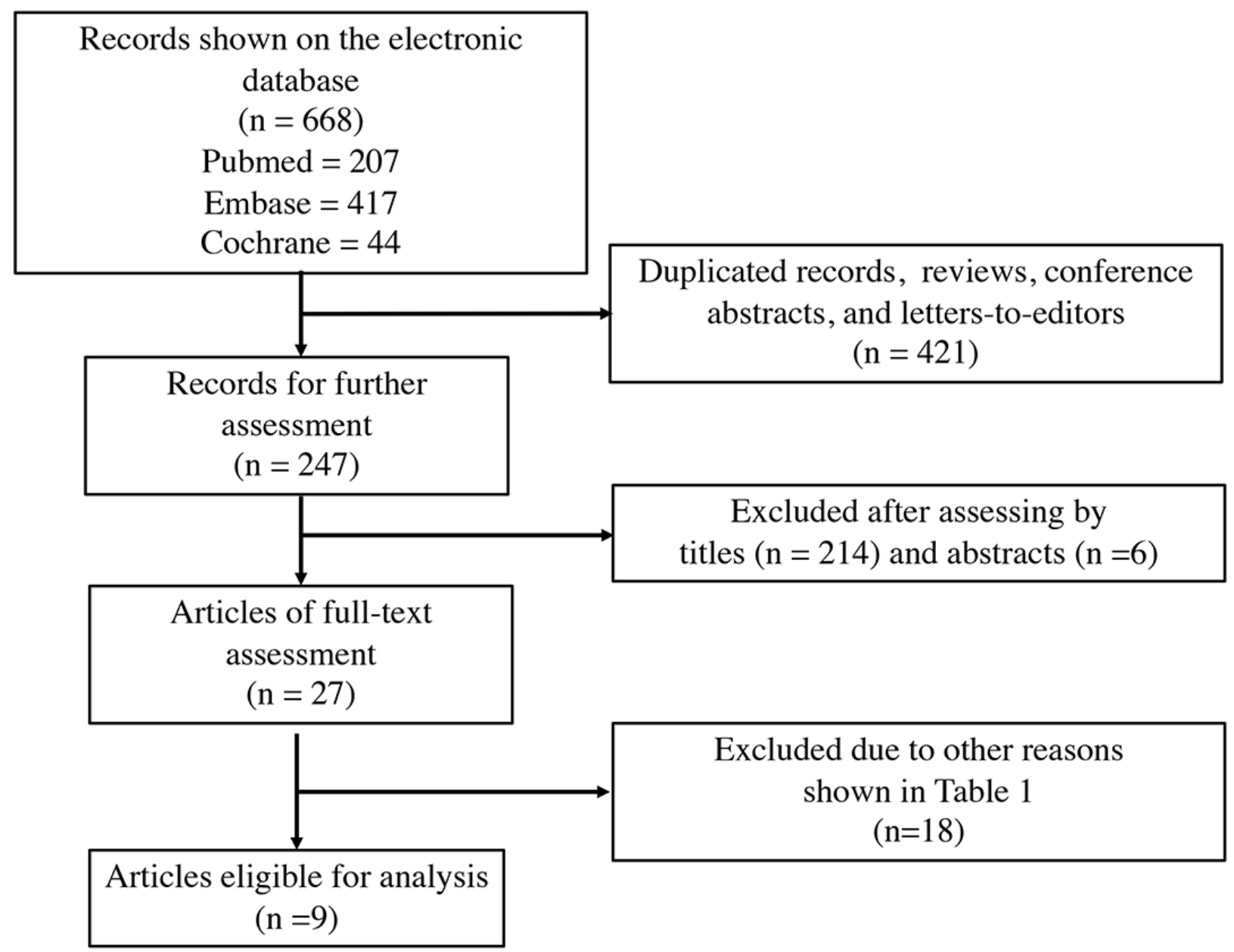

Figure 1

Flowchart for study selection 
(a) Unadjusted HR for DMFS

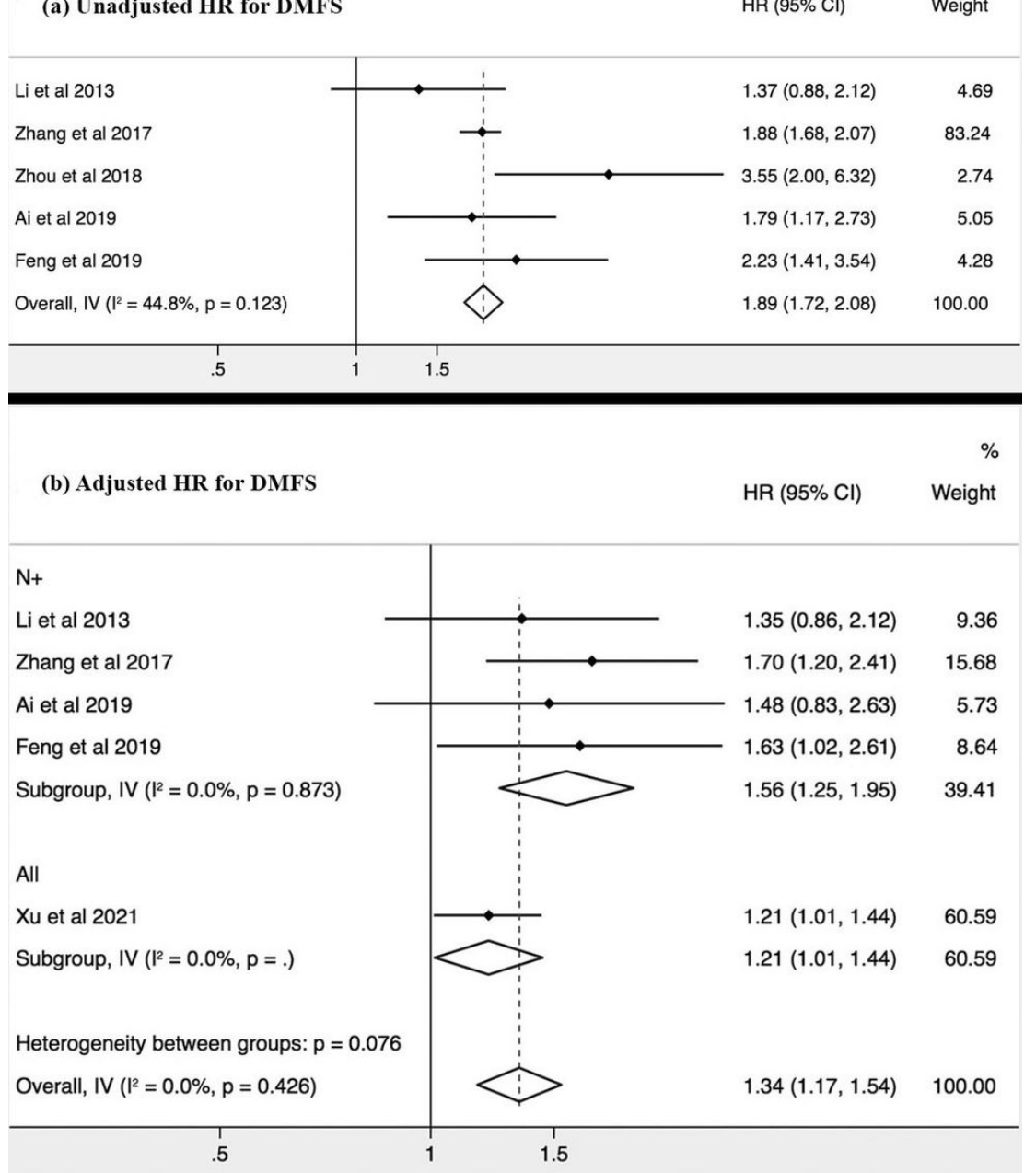

\section{Figure 2}

Forest plots of the meta-analysis showing the pooled hazard ratios (HRs) of CNN presence in any nodal group for distant metastases free survival (DMFS). Meta-analysis was performed for the unadjusted HRs (a) and adjusted HRs (b), respectively. 
(a) Unadjusted HR for DFS

$\mathrm{HR}(95 \% \mathrm{Cl})$

Weight

Li et al 2013

Zhang et al 2017

Overall, $\mathrm{DL}\left(\mathrm{I}^{2}=71.1 \%, \mathrm{p}=0.063\right)$

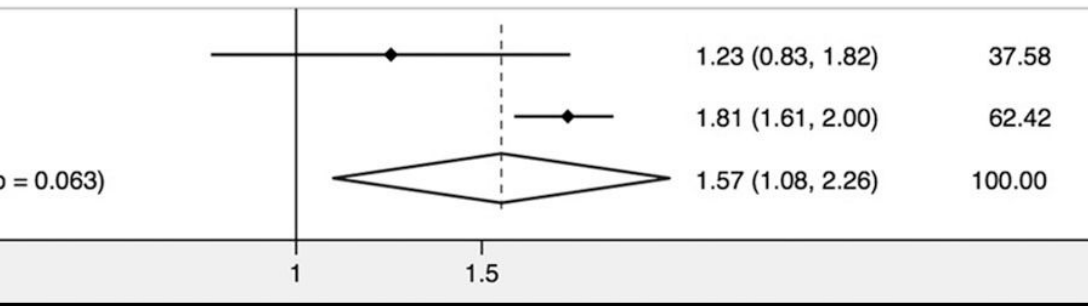

.5

(b) Adjusted HR for DFS

HR $(95 \% \mathrm{Cl}) \quad$ Weight

$\mathrm{N}+$

Li et al 2013

Zhang et al 2017

Subgroup, IV $\left(I^{2}=40.1 \%, p=0.196\right)$

All

Xu et al 2021

Subgroup, IV $\left(I^{2}=0.0 \%, p=.\right)$

Heterogeneity between groups: $p=0.431$

Overall, IV $\left(I^{2}=12.7 \%, p=0.318\right)$

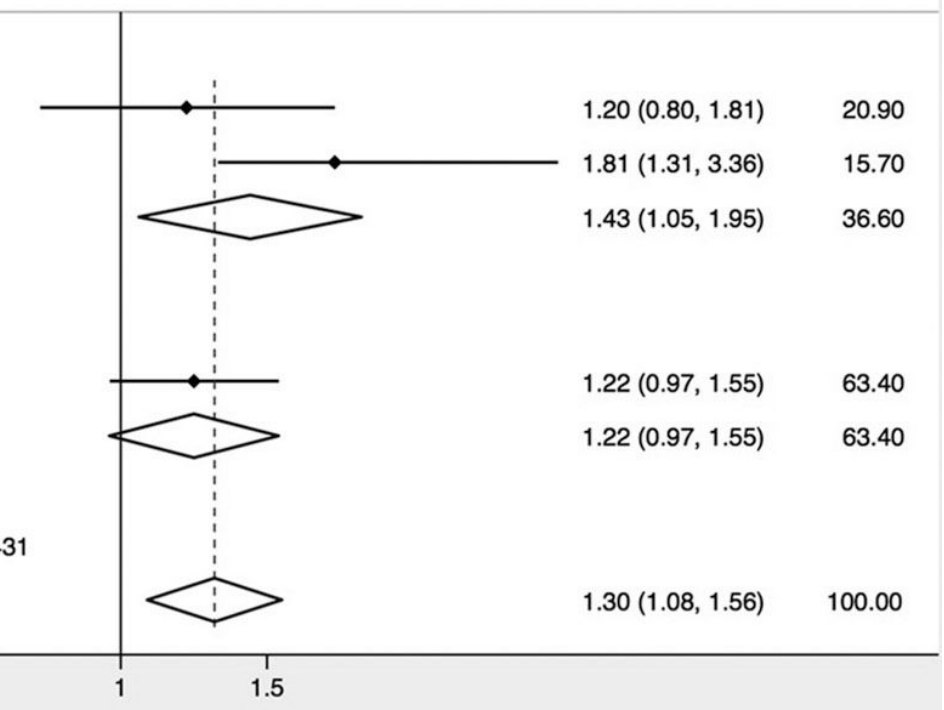

Figure 3

Forest plots of the meta-analysis showing the pooled hazard ratios (HRs) of CNN presence in any nodal group for disease free survival (DFS). Meta-analysis was performed for the unadjusted HRs (a) and adjusted HRs (b), respectively 
(a) Unadjusted HR for OS

$\mathrm{HR}(95 \% \mathrm{Cl}) \quad$ Weight

\begin{tabular}{|c|c|c|c|}
\hline Zhang et al 2017 & $\rightarrow$ & $1.87(1.67,2.06)$ & 88.27 \\
\hline Ai et al 2019 & - & $1.60(1.13,2.25)$ & 8.20 \\
\hline Feng et al 2019 & & $2.57(1.52,4.34)$ & 3.53 \\
\hline Overall, IV $\left(I^{2}=9.1 \%, p=0.333\right)$ & & $1.87(1.69,2.06)$ & 100.00 \\
\hline .5 & 1.5 & & \\
\hline
\end{tabular}

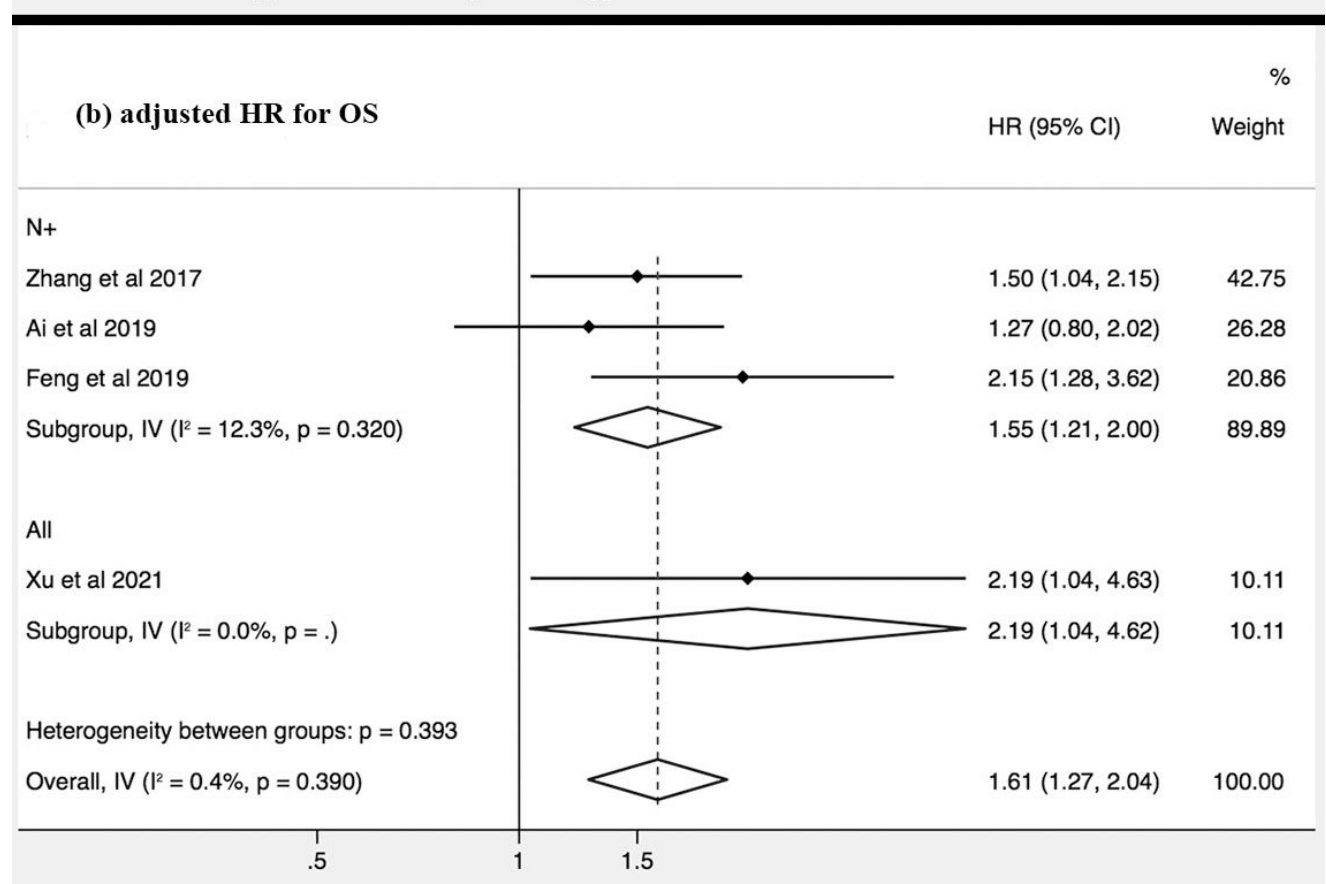

\section{Figure 4}

Forest plots of the meta-analysis showing the pooled hazard ratios (HRs) of CNN presence in any nodal group for overall survival (OS). Meta-analysis was performed for the unadjusted HRs (a) and adjusted HRs (b), respectively. 


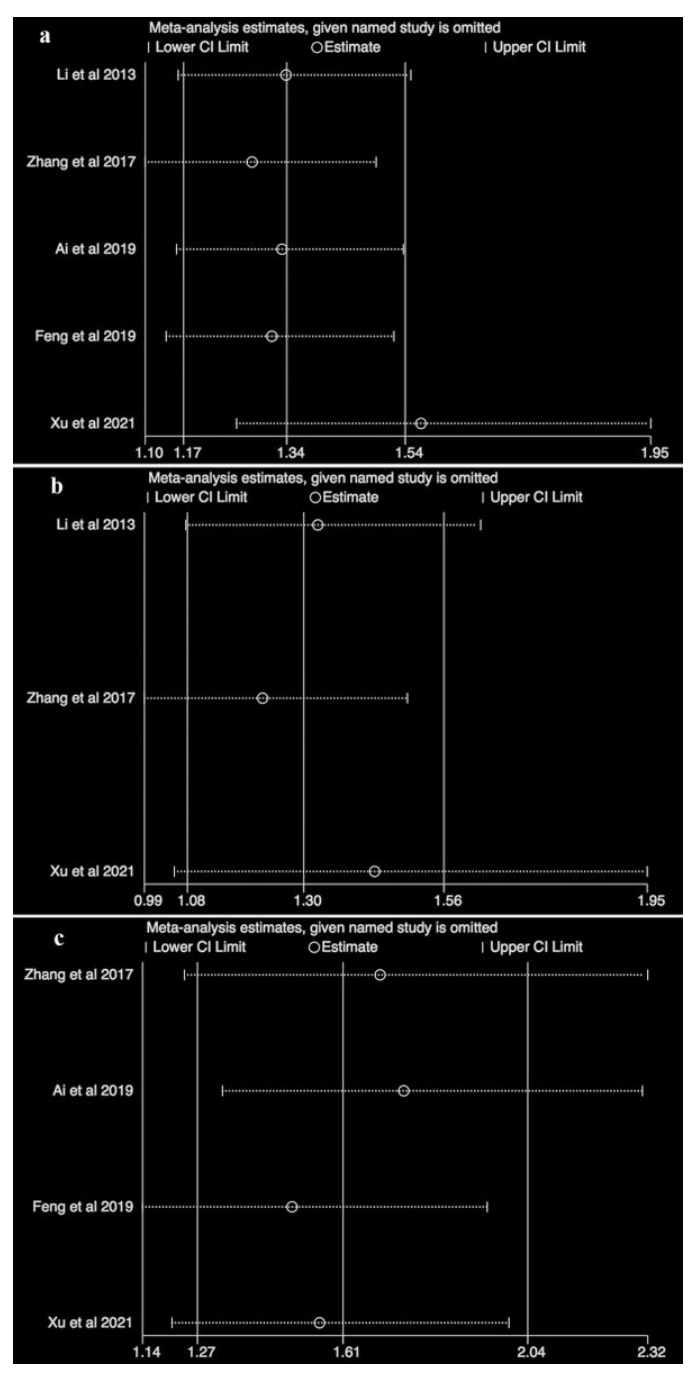

\section{Figure 5}

Sensitivity analysis showing the association between the adjusted hazard ratios (HRs) of CNN presence in any nodal group and the distant metastases-free survival (a), disease-free survival (b) and overall survival (c). 